\title{
Mushroom Microlenses: Optimized Microlenses by Reflow of Multiple Layers of Photoresist
}

\author{
P. Heremans, Member, IEEE, J. Genoe, M. Kuijk, Member, IEEE, R. Vounckx, Member, IEEE, and G. Borghs
}

\begin{abstract}
A novel type of photoresist microlenses is conceived. The characteristic of these lenses is their mushroom shape: the lens dome is located at a certain controllable distance from the substrate, and the curvature of the lens is larger than that of traditional truncated-sphere lenses. These mushroom microlenses are equilibrium-state, stable products of reflow that are obtained when starting from a multilayer photoresist stack. Integration on light-emitting diodes results in enhanced output efficiency and directivity: a vertical-to-surface emission enhancement of up to a factor of 8 has been achieved.
\end{abstract}

Index Terms - Lenses, light-emitting diodes, resists.

\section{INTRODUCTION}

$\mathbf{M}$ ICROLENSES with diameters of a few micrometers to a few hundred micrometers are used to boost the efficiency of semiconductor optical devices such as detectors and emitters. When combined to emitters, they can enhance the total light output or redirect or reshape the light output pattern. A popular technique to fabricate microlenses is the photoresist reflow technique [1]. A single-layer circular photoresist island is heated to above the reflow temperature, thereby allowing the resist to draw into a truncated-sphere shape, and then solidified by cooling down. The resulting lens is a planoconvex spherical lens characterized by a radius $R$, a height $H$ (maximum distance between the two lens surfaces), a focus distance $f$ and an index of refraction $n$. The height of such lenses is always smaller than half their radius, unless they are fabricated on a pedestal [2]. The focus distance is related to the lens radius [3] as

$$
f=\frac{n}{n-1} R
$$

For photoresist, $\mathrm{n} \approx 1.6$, hence the focal distance is about 2.6 times the lens radius. For efficient operation, the active semiconductor device should be located at the focus distance of the lens. This means that either the lens must be fabricated on a substrate like a glass plate and kept at focus distance from the active semiconductor device, or the lens must be made on a pedestal [2] on the active device.

In this letter, we propose and evaluate a new microlensforming technique based on reflow of multiple photoresist lay-

Manuscript received April 21, 1997. The work J. Genoe was supported by the Geconcerteerde Onderzoeksaktie. The work of M. Kuijk was supported by the Belgian NFWO under a Post-Doctoral grant.

P. Heremans, J. Genoe, M. Kuijk, and G. Borghs are with IMEC, B-3001 Leuven, Belgium.

J. Genoe, M. Kuijk, and R. Vounckx are with ETRO, VUB, B-1050 Brussels, Belgium.

Publisher Item Identifier S 1041-1135(97)06394-5.
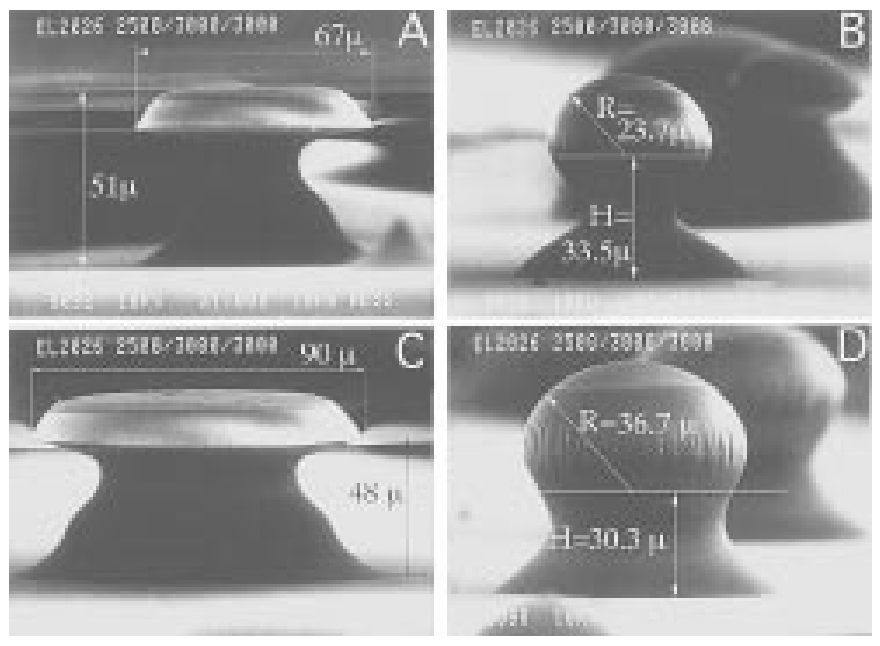

Fig. 1. Resist profiles after development before (left: A,C) and after (right: $\mathrm{B}, \mathrm{D})$ the reflow. The clear convex curvature is obtained in the pillar forming resin.

ers. They are more suited for integration with active devices, and have better optical characteristics than the photoresist lenses described so far.

\section{MiCROLENS FABRICATION}

For the fabrication of our microlenses, we use a viscous photoresist (Morton EL2026), which typically results in a $15-20-\mu \mathrm{m}$ layer when spun at 2500-3000 rpm. The microlenses can be formed on a variety of substrates, including semiconductors or glass, and can be aligned on top of devices, which are already present in these substrates. The process consists of depositing several layers of the photoresist. Only the top layer is patterned using a mask, which is eventually aligned to underlying semiconductor devices. In more detail, a typical lens-forming process is as follows. A first layer of photoresist is spin coated and prebaked, then uniformly flooded with proper UV light and dried. This drying process is required for the layer to withstand the prebake step of the subsequent layer. It is typically performed at moderate temperatures $\left(30^{\circ} \mathrm{C}-40{ }^{\circ} \mathrm{C}\right)$ for a long period of time $(24 \mathrm{~h})$. Next, the process of spin coating, prebake, flood and dry is optionally repeated for a second photoresist layer. Then, a final layer of photoresist is spun, prebaked and illuminated through a mask. The total photoresist structure is now developed. During this process, the top resist layer is structured according to the mask, and serves as mask for the underlying resist layers which were illuminated uniformly. Typical structures 
obtained after development are shown in Fig. 1(A) (diameter $67 \mu \mathrm{m}$ ) and Fig. 1(C) (diameter $90 \mu \mathrm{m}$ ). When these structures are reflowed at $100{ }^{\circ} \mathrm{C}$ for $4 \mathrm{~min}$, the final lens shape of Fig. 1(B) and (D) are obtained. They are characterized by a typical mushroom shape. It is readily observed that the focus of the lens of Fig. 1(B) is close to the semiconductor surface, where the light of the diode is emitted. An almost parallel light output beam is, therefore, expected, which is experimentally confirmed in Section IV.

\section{CAlculated Lens ShaPes}

When the photoresist structure is brought to reflow temperature, it is reshaped by the surface tension. This surface tension is a material constant. We consider that our lenses consist of two types of materials: illuminated photoresist in the lower region, originating from the layers which were flooded uniformly with light and dried extensively, and nonilluminated photoresist in the upper region. We assume that the volume of these photoresist types remain constant during reflow. Furthermore, because the photoresist is above the reflow temperature, we assume that the internal pressure is a constant, apart from the small influence of the gravity, which we also take into account. Finally, because we are primarily interested in lenses formed with a circular mask, we limit our analysis to surfaces of revolution, obtained by rotating a curve with arc length $l$ around a central axis. With these restrictions, the shape of the lens after reflow is a socalled Delaunay surface. The differential equation describing the relation between the surface tension $(\lambda(l))$, the internal pressure $(p)$, and the geometry of such structures is [4]:

$$
\frac{d^{2} r(l) / d l^{2}}{d h(l) / d l}-\frac{d h(l) / d l}{r(l)}=\frac{p}{\lambda(l)}
$$

with $h$ the height above the substrate. By solving (2) numerically, using the boundary conditions for the pressure and volumes described above, we calculate the shape of lenses corresponding to different internal pressures. Fig. 2(A)-(I) shows the results.

First, we observe that mushroom-shapes are indeed possible. This may seem counter-intuitive, because the gravity (that we take into account) is expected to spread the photoresist to the truncated-sphere shape. The calculations show that for mushroom shapes to be possible, it is required that the ratio $p / \lambda$ of the bottom part is smaller than that of the top part. From the experimentally observed mushroom shapes, we conclude that the surface tension of the illuminated photoresist is higher than that of the nonilluminated photoresist. All shapes of Fig. 2(A)-(I) were obtained assuming a ratio of between the surface tension of the bottom part to that of the top part of 1.2.

Each lens shape shown in Fig. 2(A)-(I) is characterized by a certain surface tension energy. The relationship between this surface tension energy and the internal pressure is also shown in Fig. 2. It is clear that among all the shapes shown in Fig. 2, only those shapes corresponding to a local minimum of the surface tension energy are possible equilibrium shapes.

It can be seen that there is one shape which results in a maximum surface tension energy: shape E. Starting from this
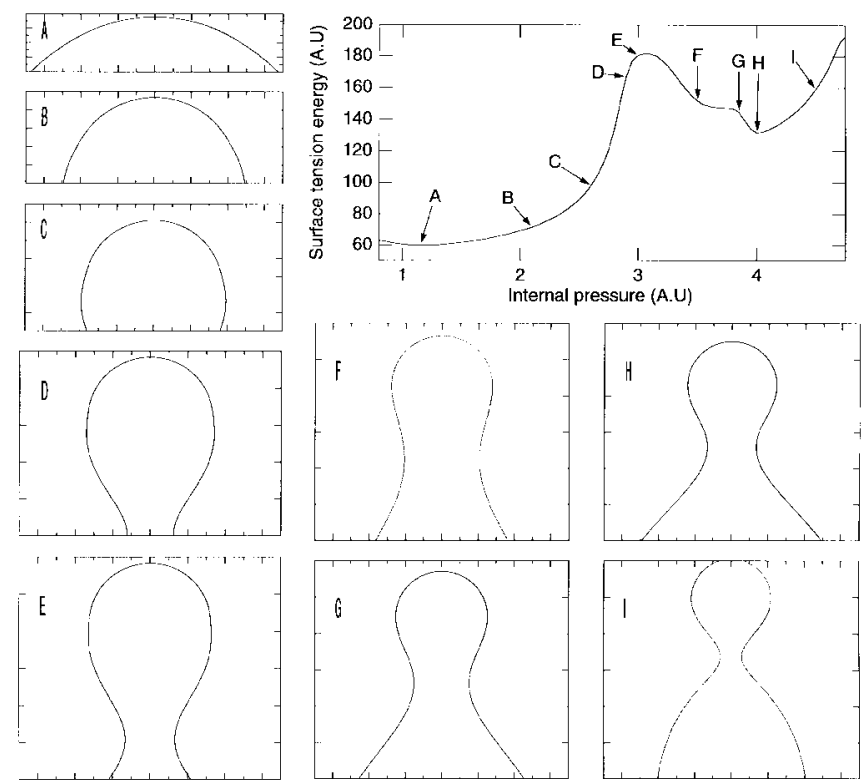

Fig. 2. Lens shapes for different relative internal pressures showing both the evolutions toward a mushroom shape $(\mathrm{H})$ or traditional shape $(\mathrm{A})$. The upper figure on the right hand side shows the total surface tension energy as a function of the internal pressure. Two local minima in energy are observed.

shape, the lens will obviously deform by reflow to a shape with lower surface tension energy. This can occur in two directions. Either the lens sags (trajectory: shapes D,C,B,A) to the truncated-sphere shape traditionally observed, or it is pulled into a mushroom shape (trajectory: shapes F,G,H). If the lens sags, the internal pressure drops continuously. It would become zero for a resist layer spread out completely as a thin film, were it not that the spreading stops when the contact angle with the substrate material reaches a predetermined materialrelated value $\left(35^{\circ}\right)$, which fits with the minimum A of the total surface tension energy. On this trajectory toward the minimum surface energy, one passes the shape of minimal footprint (D) and maximum contact angle (C).

The second way to evolve starting from shape $\mathrm{E}$ is to a clearly defined mushroom shape. In this case, too, the contact angle, the height and the energy decrease from shape $\mathrm{E}$ to shape $\mathrm{H}$. The footprint increases continuously in this region. From Fig. 2, it is clear that the starting condition of the reflow process determines to which of these equilibrium states the lens evolves. ${ }^{1}$ The mushroom shape has a higher internal pressure than the truncated-sphere shape, but as it corresponds to a local energy minimum, it remains stable. Indeed, for the lens to evolve quasistatically from the mushroom shape to the truncated-sphere shape, both the energy and the contact angle must go over a maximum. Mushroom microlenses are therefore stable on light-emitting devices that heat during operation.

\section{EXPERIMENTAL INTEGRATION WITH LEDS}

We formed an array of microlenses (Fig. 3) on top of an array of light-emitting diodes and measured the LED emission profiles before and after integration with the microlenses.

\footnotetext{
${ }^{1}$ Calculations of the evolution of the lens shape as a function of reflow time will be published elsewhere.
} 


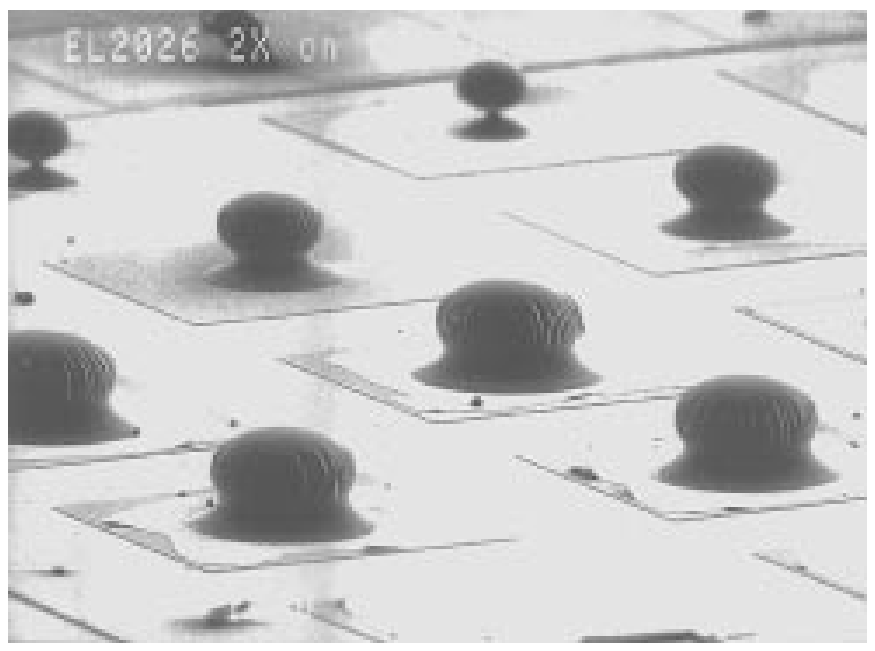

Fig. 3. An array of lenses on an array of light-emitting diodes.
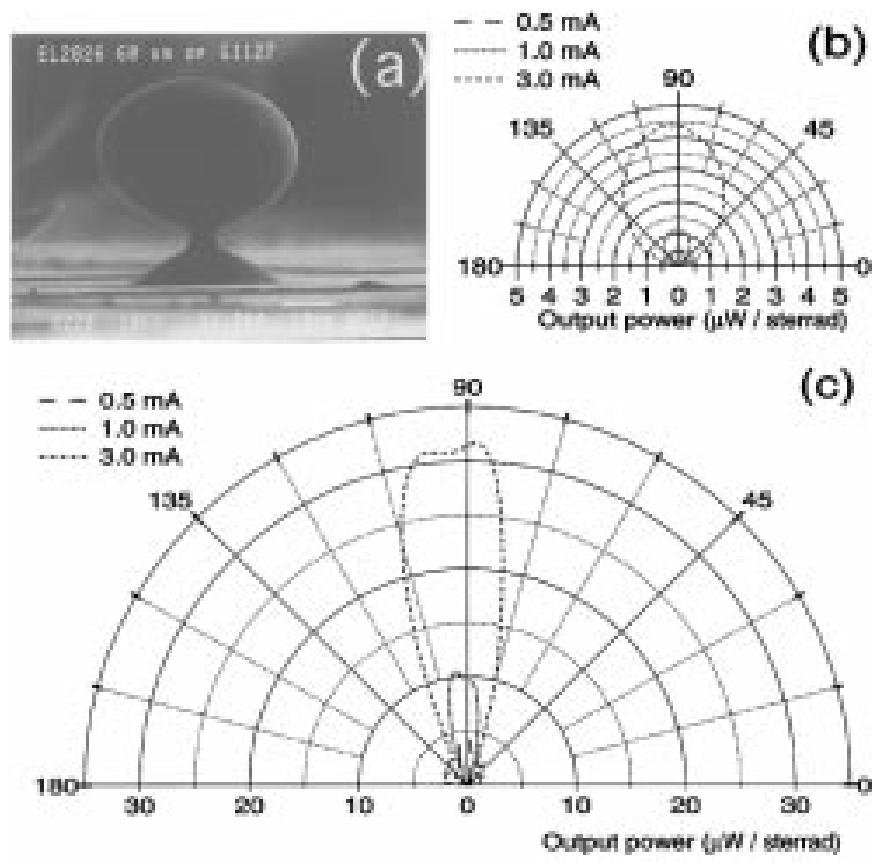

Fig. 4. Polar diagram of the measured output power of the $20-\mu \mathrm{m}$ LED without (b) and with (c) the lens shown in (a). This lens is placed on the smallest LED shown in the array of Fig. 3(center, top).

Optimum performance could be achieved with the lens shown in Fig. 4(a), combined with a $20-\mu \mathrm{m}$ diameter LED. Comparing the polar diagrams before [Fig. 4(b)] and after [Fig. 4(c)] integration with the microlens shows that due to the presence of the microlens the output power increases in the direction perpendicular to the surface by a factor of 8 and

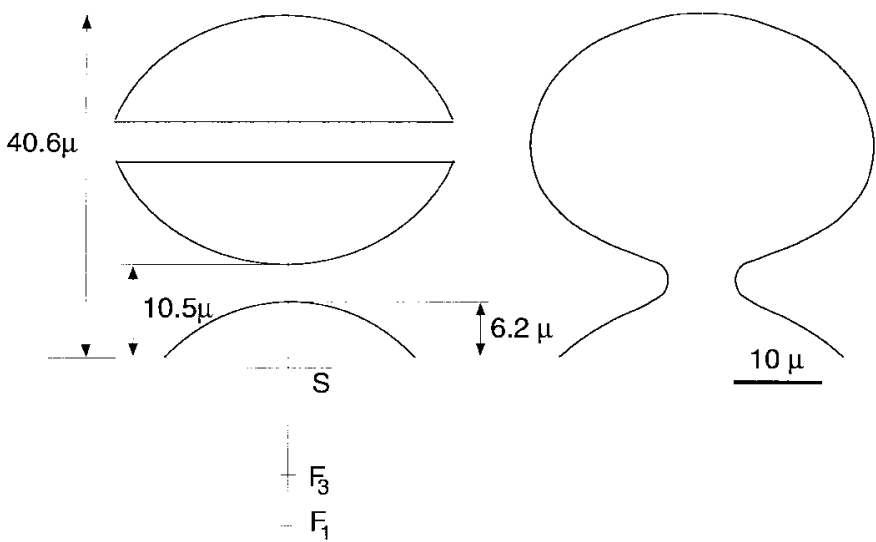

Fig. 5. Lens system equivalent to the lens shown in Fig. 4(a).

that the total integrated power increases by a factor of 2 . A closer analysis of the lens of Fig. 4(a) reveals that it performs as a three-lens system rather than as a single lens. The three lenses are shown in Fig. 5. The upper lens is located at $40 \mu \mathrm{m}$ from the semiconductor surface, and has its focus $F_{1} 20 \mu \mathrm{m}$ below the substrate surface, while the actual light source $(S)$ is less than $1 \mu \mathrm{m}$ below the surface. The lower two lenses shift the actual focus $\left(F_{3}\right) 6 \mu \mathrm{m}$ higher, such that it lies at $13 \mu \mathrm{m}$ from the light source. This explains the very good collimation of the output light beam by the lensset.

\section{CONCLusion}

We report on a novel type of microlenses: the mushroom microlenses. They are shown to be a stable product of reflow of a stack of photoresist layers, and have two appealing characteristics: their curvature is stronger than that of conventional truncated-sphere photoresist microlenses, and the distance of the lens to the substrate is controllable. These characteristics permit to optimize the microlens shape for specific applications in combination with light emitters and detectors. When combined with LED's, for instance, they can be used to maximize the directivity of the output beam, to optimize the external quantum efficiency, or to focus the light on a spot at a certain distance.

\section{REFERENCES}

[1] M. Hutley, R. Stevens, and D. Daly, "Microlens arrays," Physics World, vol. 4, no. 7, p. 27, July 1991.

[2] Z. D. Popovic, R. A. Sprague, and G. A. Neville Connell, "Technique for monolithic fabrication of microlens arrays," Appl. Opt., vol. 27, p. 1281, 1988.

[3] M. Klein, Optics. New York: Wiley, 1986

[4] M.P. Do Carmo, Differential Geometry of Curves and Surfaces. Englewood Cliffs, NJ: Prentice-Hall, 1976. 